\title{
Attenuating the effects of different levels of processing: The role of cue position and cue/word interval
}

\author{
DAVID G. ELMES and JOSEPH B.THOMPSON \\ Washington and Lee University, Lexington, Virginia 24450
}

\begin{abstract}
Within a list of words presented under incidental learning instructions, there were processing cues that signaled either semantic (pleasantness rating) or structural (syllable counting) processing of the words. Cue position (before or after the words) and cue/word interval (.5 sec or 2 sec) were varied orthogonally between subjects. Free recall was higher for words given semantic judgments than for those given structural processing except when the processing cues followed the words by 2 sec. The results indicate that if given the opportunity subjects may process the words in more than one way. Thereby, the difference in recall between "deep" and "shallow" processed words is attenuated.
\end{abstract}

Craik and Lockhart (1972) proposed that retention of a word should increase with the depth to which that word is processed. Support for this idea comes from studies, such as one by Walsh and Jenkins (1973), that show "shallow" processing involving structural judgments (e.g., counting the number of syllables) leads to poorer retention than does "deeper" processing involving semantic judgments (e.g., rating a word for its pleasantness).

Numerous tests of the levels of processing theory have used cues or instructions to inform the subjects how they should process the task materials. In withinlists designs, wherein successive items receive a cue that signals one of several different types of processing, the processing cues have occurred both before (e.g., Craik \& Tulving, 1975; Elmes \& Bjork, 1975) and after (Klein \& Saltz, 1976; Till \& Jenkins, 1973) the to-beremembered words. Moreover, the cue/word interval has taken on various values: The cues have preceded the words by as much as $2 \mathrm{sec}$ and have followed the words by as much as $.5 \mathrm{sec}$.

A modest extension of the levels of processing theory suggests that variations in retention due to different processing cues in a within-lists design should be determined by whether the cues precede or follow the words and by the cue/word interval. When a cue precedes a word, the subject has a single processing command to obey on the upcoming word. In terms of the speed/accuracy instructions usually given in these situations, it is to the subject's advantage to simply perform with alacrity the single judgment required of him when the word appears. In contrast to this, when a cue follows the word, the subject may anticipate the cue by engaging in multiple processing activities. After the word is presented, the subject must wait until the cue appears so that he can make an overt response. Thus, there is a good chance for more than one type of proces- sing, and this opportunity for multiple processing should increase as the interval between the word and the cue becomes longer. Obviously, if a subject were to process many words both structurally and semantically, it would be difficult to assess the relative effects of these two types of processing on subsequent retention.

Since multiple processing may occur in the withinlists paradigm, we decided to test for this possibility directly by comparing the effectiveness of processing cues presented before and after target words with long and short cue/word intervals. We examined the incidental memory for words preceded or followed by semantic (pleasantness rating) or structural (syllable counting) cues by either $.5 \mathrm{sec}$ or $2 \mathrm{sec}$. Pleasantness ratings should yield better recall than syllable counting, and if multiple processing occurs the difference in recall between these two tasks should be smallest when the cues follow the words by $2 \mathrm{sec}$.

\section{METHOD}

\section{Subjects}

Seventy-two male introductory psychology students at Washington and Lee University were equally divided among four between-subjects conditions. These independent groups represented the factorial combination of two positions of the processing cues (before and after the target words) and two cue/ word intervals $(.5 \mathrm{sec}$ and $2 \mathrm{sec})$.

\section{Materials and List Design}

The list of 36 target words contained equal numbers of 1-, 2-, and 3-syllable words, which were chosen so that onethird of each length were judged by us to be neutral in connotation (e.g., link, volume, and ensemble), one-third were judged to be pleasant (e.g., charm, humor, and salary), and the remaining third were deemed to be unpleasant (e.g., gore, demon, and pollution). All targets had moderate imagery values $(\mathrm{M}=4.59, \mathrm{R}=4.2-5)$ according to the Paivio, Yuille, and Madigan (1968) norms. Six additional words chosen from the same source served as buffers: three each at the beginning and 
end of the list. A buzzer and a bell of about .5-sec duration served as the processing cues.

The 42 words ( 36 targets and 6 buffers) and the processing cues were recorded on magnetic tape. Four tapes were prepared, each representing one of the four orthogonal combinations of two cue positions (before and after the words) and two cue/ word intervals (.5 sec and $2 \mathrm{sec}$ ). Half the words were preceded (or followed) by a buzzer and half were preceded (or followed) by a bell. On all tapes the words occurred in the same order, and each word was always paired with the same signal. Order of type of target words and their processing cues within the list was determined by block randomization so that each half of the list contained all combinations of syllable number (three), pleasantness (three), and type of processing cue (two). Thus, these 18 combinations occurred twice within the list, yielding the 36 target words.

\section{Procedure}

A combination of mimeographed and taped instructions were given to groups of 18 subjects who then heard one of the four test tapes. Nine subjects in each group received written instructions to give pleasantness ratings when they heard the buzzer and to count syllables when they heard the bell. The written instructions reversed the cue-processing task relationship for the other nine subjects in each group, thereby assuring that words were counterbalanced across processing tasks in each group.

Numbered blank spaces appeared on each instruction sheet for noting the processing judgments. As the words and processing cues were presented, the subjects wrote down 1,2 , or 3 according to the number of syllables in the word when they heard one cue, and they wrote down $\mathrm{P}, \mathrm{U}$, or $\mathrm{N}$ for the judged pleasantness, unpleasantness, or neutrality of the word when they heard the other cue. Regardless of the cue/word interval or the position of the cue, the subjects had $3 \mathrm{sec}$ to write down their processing judgments, and they were instructed to perform the processing tasks quickly and accurately so that materials could be selected for subsequent human learning experiments. Following the final buffer word and without forewarning, the subjects were asked to write down as many of the words as possible. Unlimited time was allowed for this free recall test.

\section{RESULTS $^{1}$}

\section{Word Characteristics}

According to Duncan's Multiple Range Test, the proportions of pleasant words $(M=.18)$ and unpleasant words $(\mathrm{M}=.21)$ recalled did not differ, and both were recalled better than neutral words $(M=.14)$. Similar proportions of one-syllable $(M=.19)$ and three-syllable $(M=.20)$ words were recalled, and both were recalled better than two-syllable words $(M=.13)$. Other effects of number of syllables and pleasantness were neither systematic nor reliable.

\section{Cue Position, Cue/Word Interval, Type of Processing}

Shown in Figure 1 are the proportions of words recalled in the conditions of major interest. There was marked superiority of recall of rated words over counted words $[F(1,68)=90.93, \mathrm{MSe}=2.13]$, and there were no simple effects due to cue position and cue/word interval. These results must be considered in the context of two interactions apparent in Figure 1. Recall after syllable

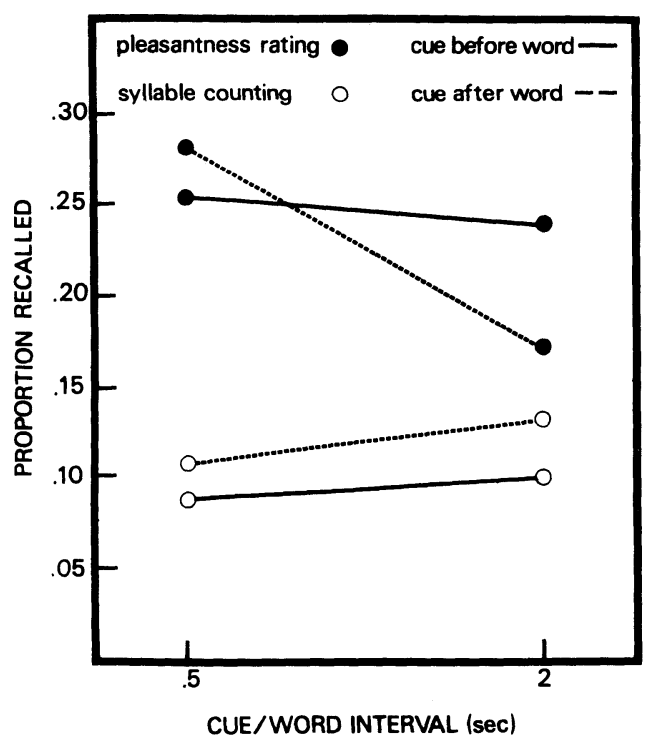

Figure 1. Proportion of words free recalled as a function of cue/word interval.

counting was slightly higher at the 2 -sec cue/word interval than at the .5 -sec interval, but the recall of rated words was much poorer at the longer than at the shorter interval $[F(1,68)=8.48]$. In the second interaction, the smallest difference in recall between the two types of processing occurred when the processing cues followed the words by $2 \sec [F(1,68)=4.46]$. A Duncan's test showed that recall of rated words when the cue followed the word by $2 \mathrm{sec}$ was lower than recall in any other rating condition, and differed from recall in the syllable counting conditions, except when the cue to count followed the target by $2 \mathrm{sec}$.

\section{DISCUSSION}

The present results demonstrate that the smallest effect of type of processing on retention occurs when the processing cue follows the word by $2 \mathrm{sec}$. This is consistent with the hypothesis that, given enough time, the subject will engage in more than one type of processing when the cue follows the word. Apparently, when the cue follows the word by $2 \mathrm{sec}$, the subjects attempted to anticipate the processing cue by covertly making both judgments (counting the syllables and rating for pleasantness) before they had to make their overt response. Because this multiple processing attenuated the difference in the level of recall following semantic and structural processing cues, it would behoove investigators interested in levels of processing to avoid presenting the processing cues long after the target words have been presented.

An additional aspect of the present results that argues against the use of delayed after-cues is the fact that recall of words rated for pleasantness was significantly worse when the cue occurred $2 \mathrm{sec}$ after the targets than in any other rating condition. This result is directly contrary to the idea that the subjects elaboratively rehearse the words while they are waiting for the cue. Instead, this finding indicates that the subjects make their judgments and then hold these judgments in memory, while they await the cue that specifies which judgment they are to 
write down. Thus, the subjects may be rehearsing information that is inappropriate for the purposes of a particular experiment. This possibility is testable, since one could measure recall of processing judgments given to the words. According to our hypothesis, recall of the processing judgments should be least accurate for judgments signaled by delayed cues. Alternatively, if the subjects were faced with several possible processing tasks, each of which required substantial processing capacity, a delayed cue might enhance item recall since the subjects would, in this situation, rehearse the word rather than engage in multiple processing.

It should be noted that our results do not seem to agree with those of Walsh and Jenkins (1973). They used a betweensubjects design and presented processing instructions prior to list presentation. Some of their conditions included different groups performing either semantic, structural, or combined semantic and structural processing on the words. In general, they found better recall following semantic processing than after combined semantic and structural judgments. However, only one of seven contrasts yielded a significant decrement in recall with the combined semantic and structural tasks. Walsh and Jenkins argued that their results are consistent with a depth of processing view and inconsistent with the ideas that effort or difficulty of processing are crucial. Since our results show a marked decrement in recall under a condition where the subject is likely to be engaging in both semantic and structural processing, it could be argued that our results go against the depth of processing hypothesis and the results reported by Walsh and Jenkins.

On the contrary, we believe that our results are completely consistent with both the depth of processing view and the data of Walsh and Jenkins. Our point is that processing cues presented before the targets place different task demands on the subject than do cues presented after the words. In the latter case, but not in the former, the subject may make anticipatory judgments and rehearse those judgments. On the other hand, when cues precede the words and the subjects are obliged to make two judgments under time stress, as in the Walsh and Jenkins study, it is likely that there would be some slight decrement in performance. In any event, we agree (and our data demonstrate) that how subjects process an item is important, and we would like to reiterate the hardly original point that what they rehearse also will determine recall.

\section{REFERENCES}

CRAIK, F. I. M., \& Lockhart, R. S. Levels of processing: A framework for memory research. Journal of Verbal Learning and Verbal Behavior, 1972, 11, 671-684.

Craik, F. I. M., \& Tulving, E. Depth of processing and the retention of words in episodic memory. Journal of Experimental Psychology: General, 1975, 104, 268-294.

Elmes, D. G., \& BjoRK, R. A. The interaction of encoding and rehearsal processes in the recall of repeated and nonrepeated items. Journal of Verbal Learning and Verbal Behavior, 1975, 14, 30-42.

KLEIN, K., \& SALTz, E. Specifying the mechanisms in a levels-of-processing approach to memory. Journal of Experimental Psychology: Human Learning and Memory, 1976, 2, 671-679.

Paivio, A., Yuille, J. C., \& Madigan, S. A. Concreteness, imagery, and meaningfulness values for 925 nouns. Journal of Experimental Psychology Monograph, 1968, 76 (1, Part 2).

Till, R. E., \& Jenkins, J. J. The effects of cued orienting tasks on the free recall of words. Journal of Verbal Learning and Verbal Behavior, 1973, 12, 489-498.

Walsh, D. A., \& Jenkins, J. J. Effects of orienting tasks on free recall in incidental learning: "Difficulty," "effort," and "process" explanations. Journal of Verbal Learning and Verbal Behavior, 1973, 12, 481-488.

\section{NOTE}

1. The significance level chosen was $p<.05$.

(Received for publication April 11, 1977.) 